\title{
BMJ Open Eleven-year multimorbidity burden among 637255 people with and without type 2 diabetes: a population-based study using primary care and linked hospitalisation data
}

To cite: Zghebi SS, Steinke DT, Rutter MK, et al. Elevenyear multimorbidity burden among 637255 people with and without type 2 diabetes: a population-based study using primary care and linked hospitalisation data. BMJ Open 2020;10:e033866. doi:10.1136/ bmjopen-2019-033866

- Prepublication history and additional material for this paper are available online. To view these files, please visit the journal online (http://dx.doi. org/10.1136/bmjopen-2019033866).

Received 25 August 2019 Revised 01 May 2020 Accepted 05 May 2020
D) Check for updates

(C) Author(s) (or their employer(s)) 2020. Re-use permitted under CC BY-NC. No commercial re-use. See rights and permissions. Published by BMJ.

For numbered affiliations see end of article.

Correspondence to Dr Salwa S Zghebi; salwa.zghebi@manchester. ac.uk

\section{ABSTRACT}

Objectives To compare the patterns of 18 physical and mental health comorbidities between people with recently diagnosed type 2 diabetes (T2D) and people without diabetes and how these change by age, gender and deprivation over time between 2004 and 2014. Also, to develop a metric to identify most prevalent comorbidities in people with $\mathrm{T} 2 \mathrm{D}$.

Design Population-based cohort study.

Setting Primary and secondary care, England, UK.

Participants 108588 people with T2D and 528667 comparators registered in 391 English general practices. Each patient with T2D aged $\geq 16$ years between January 2004 and December 2014 registered in Clinical Practice Research Datalink GOLD practices was matched to up to five comparators without diabetes on age, gender and general practice.

Primary and secondary outcome measures Prevalence of 18 physical and mental health comorbidities in people with T2D and comparators categorised by age, gender and deprivation. Odds for association between T2D diagnosis and comorbidities versus comparators. A metric for comorbidities with prevalence of $\geq 5 \%$ and/or odds $\geq 2$ in patients with T2D.

Results Overall, $77 \%$ of patients with T2D had $\geq 1$ comorbidity and all comorbidities were more prevalent in patients with T2D than in comparators. Across both groups, prevalence rates were higher in older people, women and those most socially deprived. Conditional logistic regression models fitted to estimate $(0 \mathrm{R}, 95 \% \mathrm{Cl})$ for association between T2D diagnosis and comorbidities showed that T2D diagnosis was significantly associated with higher odds for all conditions including myocardial infarction (OR 2.13, 95\% $\mathrm{Cl} 1.85$ to 2.46 ); heart failure (OR $2.12,1.84$ to 2.43); depression (OR 1.75, 1.62 to 1.89 ), but non-significant for cancer (OR 1.12, 0.98 to 1.28). In addition to cardiovascular disease, the metric identified osteoarthritis, hypothyroidism, anxiety, schizophrenia and respiratory conditions as highly prevalent comorbidities in people with T2D.

Conclusions T2D diagnosis is associated with higher likelihood of experiencing other physical and mental illnesses. People with T2D are twice as likely to have cardiovascular disease as the general population. The findings highlight highly prevalent and under-reported
Strengths and limitations of this study

- This is the first large study examining the annual patterns of multimorbidity between people with and without type 2 diabetes (T2D) using primary care and hospitalisation data in England.

- The study examines the patterns of 18 comorbid physical and mental conditions, whereas prior research has mainly focused on patterns of cardiovascular disease in people with $\mathrm{T} 2 \mathrm{D}$ resulting in little understanding of trends of a broader range of important comorbidities in people with and without T2D.

- The study highlights high prevalence of comorbidities associated with T2D diagnosis that are either under-reported or not featured in diabetes guidelines, mainly osteoarthritis, anxiety, schizophrenia and respiratory conditions.

- To minimise misclassification, prescribed treatments and time-windows were examined as additional measures to confirm the active status of five resolvable medical conditions such as depression and asthma.

- The study relies on recorded diagnoses in electronic health records, and therefore, there is a possibility to have missed patients with undiagnosed illnesses such as patients who did not consult general practice or hospital setting.

comorbidities in people with T2D. These findings can inform future research and clinical guidelines and can have important implications on healthcare resource allocation and highlight the need for more holistic clinical care for people with recently diagnosed T2D.

\section{INTRODUCTION}

Diabetes is a chronic metabolic condition associated with global economic burden and increased risks for premature mortality. ${ }^{1}$ Currently, nearly 422 million adults have diabetes worldwide. ${ }^{2}$ Previous reports have showed that people diagnosed with type 2 
diabetes (T2D) were at up to $93 \%$ significantly excess risk for mortality than people without diabetes. ${ }^{3-5}$

Improvements in life expectancy observed over recent years have resulted in an ageing population living with multiple clinical conditions. Multimorbidity is the co-occurrence of two or more chronic conditions in the same individual. ${ }^{6}$ People living with multimorbidity often have poorer quality of life and are prone to adverse outcomes. ${ }^{6}$ Characterising coexisting morbidities in the T2D populations is important as it may explain current medical utilisation patterns, highlight potential interactions with diabetes management and guide future healthcare planning.

Multimorbidity has been identified as a priority for global health research. ${ }^{7}$ Several earlier studies have described comorbidities in the T2D population, but mainly as a part of a different research aim or not in UK population or typically confined to trends of cardiovascular disease. Prior studies either based on small cohorts ${ }^{89}$; or on a subgroup of patients with T2D, for example, within a specific age category. ${ }^{10}{ }^{11}$ In addition, the examined comorbidities were mainly reported as an overall count ${ }^{9}$; or limited to diabetes-related complications. ${ }^{12}$ Overall, it is estimated that approximately $44 \%-95 \%$ of people with diabetes, including some specific diabetes cohorts such as veterans and elderly people, have one or more comorbid conditions. ${ }^{13} 14$ A Dutch study assessed the impact of comorbidities burden in people with diabetes and reported a positive correlation between the number of comorbidities and healthcare utilisation. ${ }^{14}$

Despite the importance of examining the multimorbidity burden in people with T2D, there is limited understanding on the profile of other prevalent physical and mental health illnesses in people with T2D while no previous study has characterised and compared these trends in people with and without T2D. In addition, most current clinical guidelines and strategies have 'single condition' model of care without considering multimorbidity.

In this study, we used large electronic linked primary care and hospital electronic health data to examine the patterns of 18 physical and mental health conditions in people with and without T2D over an 11-year study period in England. We further stratified the comorbidities prevalence rates by age, gender, social deprivation and assessed the association between diagnosis of T2D and the presence of a comorbidity and described the patterns of all two coexisting comorbidities. In people with T2D, we developed a metric to identify most prevalent comorbidities and examined whether these conditions were addressed in national diabetes guidelines.

\section{METHODS}

\section{Data source}

The Clinical Practice Research Datalink (CPRD) GOLD is an electronic database of medical records from general practices across the UK. ${ }^{15}$ General practice is part of primary care for patients in the UK and the gate-keeper for access to secondary and tertiary healthcare services. The CPRD provides longitudinal anonymised data on patient demographics, clinical diagnoses, prescribed treatments, tests and referrals and is broadly representative of the UK population in terms of age, gender and ethnicity. ${ }^{16}{ }^{17}$ In CPRD, clinical records are coded using Read codes (clinical terminology based on a hierarchical classification system). Nearly $75 \%$ of the contributing CPRD GOLD general practices in England are eligible for the CPRD data linkage scheme which provides access to external linked datasets and registries. ${ }^{16}$ These include hospitalisation, mortality and socioeconomic status data.

In this study, we used secondary care data from the Hospital Episode Statistics (HES), and patient-level socioeconomic status as indicated by the Index of Multiple Deprivation (IMD) 2010 quintiles for the matched patients with T2D and comparators registered in English general practices consented for the CPRD linkage scheme. The clinical diagnoses in HES are recorded using the 10th revision of the International Classification of Diseases (ICD-10) coding. The IMD, recorded at the level of patient's residential postcode, is a composite score calculated as the weighted sum of the individual indices of seven domains of deprivation: income, employment, education, finance, access to housing and services, living environment and crime. ${ }^{18}$

\section{Patient population}

Patients registered for $\geq 90$ days in an up-to-standard general practice eligible for data linkage, aged $\geq 16$ years, with a first diagnostic T2D medical code between 1 January 2004 and 31 December 2014, and who had no clinical record for type 1 diabetes were included as patients with T2D. The up-to-standard criterion indicates that the data provided by a practice are of a suitable quality for use in research, based on the continuity of data recording and mortality rate in comparison with the expected range. ${ }^{16} 17$ The index date was defined as the earliest date of T2D diagnosis. The identified cohort of patients with T2D was categorised according to the calendar year of T2D diagnosis (2004 to 2014). Within each year, up to five patients without diabetes (comparators) were identified and matched to each patient with T2D on the following variables at the T2D diagnosis date: age ( \pm 2 years difference), gender and general practice. Comparators were registered in linked English general practices and were alive at the index date. Each comparator without diabetes was assigned the index date of the matched T2D case and was followed up in the same manner. The annual matched cohorts are illustrated in online supplementary figure S1.

\section{Identification of comorbidities}

The diagnostic Read and ICD-10 codes for 18 physical and mental health morbidities were collated from previous literature examining chronic conditions ${ }^{192}$ and online resources. ${ }^{21}{ }^{22}$ All code lists (online supplementary table S1) were carefully reviewed by expert clinicians 
and pharmacists in our team for relevance to the examined population. The Read and ICD-10 codes were used to identify relevant diagnoses in primary care or during hospitalisation, respectively. Overall, the presence of comorbidities was defined as conditions recorded ever before T2D diagnosis date (index date) and up to the end of the calendar year of T2D diagnosis the cohort is contributing to between 2004 and 2014. Our method for identifying morbidities is based on the knowledge that the examined conditions are mostly chronic, that is, an ongoing condition once diagnosed. However, as 5 of the 18 conditions are resolvable (potentially transient) additional criteria were therefore used to ascertain their active status in individual patients by using prescriptions and/ or the timing of the diagnostic codes, as explained below.

The 18 examined morbidities were asthma, chronic obstructive pulmonary disease (COPD), hypothyroidism, osteoarthritis (OA), chronic kidney disease (CKD), anxiety, depression, schizophrenia, epilepsy, hypertension (HT), hyperlipidaemia, atrial fibrillation (AF), congestive heart failure (CHF), peripheral vascular disease (PVD), myocardial infarction (MI), transient ischaemic attack (TIA), stroke and cancer. These morbidities were primarily selected because are clinically relevant comorbidities in people with T2D and as, for the majority of them, the UK Quality and Outcomes Framework (QOF) entails their recording and reporting in primary care (ie, are financially incentivised conditions as per the UK general practice contract). The QOF pay for performance incentive scheme was introduced in 2004 to reward UK general practices providing high-quality care based on defined clinical indicators implementation of preventative measures. ${ }^{23}$

As some of the investigated morbidities might be resolved at a later stage, additional criteria (prescription and/or time-window) were introduced to define active disease status of these morbidities. ${ }^{24}$ These criteria were applied to 5 of the 18 examined conditions as follows: asthma (a Read/ICD-10 diagnostic code ever plus a prescription for inhaled corticosteroid and/or longacting beta-agonists over the last 12 months was required); anxiety (a diagnostic code in the last 12 months or $\geq 4$ prescriptions for anxiolytics/hypnotics over the last 12 months); depression (a diagnostic code in the last 12 months or $\geq 3$ prescriptions for antidepressants in the last 12 months (except low dose tricyclic antidepressants)); epilepsy (a diagnostic code ever plus a prescription for an antiepileptic drug in the last 12 months) and cancer (first diagnosis recorded in the last 5 years). Low-dose tricyclic antidepressants were defined as formulations with strength up to $25 \mathrm{mg}$. However, all strengths were included for amoxapine, butriptyline, clomipramine, dosulepin, doxepin, lofepramine, mianserin, protriptyline, trimipramine, viloxazine either because a medication was only indicated for treatment of depression or low doses were prescribed for depression treatment. Throughout this paper, we refer to the examined conditions as 'comorbidities' but we recognise that for comparators without diabetes these conditions are 'morbidities' when occurring on their own.

\section{Patient and public involvement}

No patients or public were involved in this study during design, conduct or analysis stages.

\section{Statistical analyses}

Statistical analyses were used to map comorbidity data obtained from CPRD GOLD primary care and hospitalisation data sources using six approaches:

1. First, annual prevalence rates and 95\% CI for each comorbidity in patients with T2D and matched comparators over the 11-year period were calculated. For each matched case and comparator (eg, cases and comparators in the matched cohort in 2004), the prevalence rate for a comorbidity in 2004 was calculated by dividing the number of T2D cases (or comparators) with a clinical record for that comorbidity in or before 2004 by the number of all cases (or comparators) contributing throughout 2004.

2. Second, the prevalence rates of comorbidities were stratified by (1) 20-year age groups, (2) gender and (3) patient-level social deprivation (IMD quintiles) for patients with T2D and matched comparator patients in the last study year (2014).

3. Third the patterns of coexisting two comorbidities were calculated and compared between people with T2D and people without diabetes. The pattern of all two coexisting comorbidities were visually illustrated using three dimensional (3-D) bubble plots. The size of the 3-D bubbles is determined by the proportion of patients with the two comorbidities and is relative in terms of other bubbles in the same plot and not between separate plots.

4. In the fourth approach, the overall and gender-specific annual prevalence rates of clustered cardiovascular disease were calculated for patients with T2D and matched comparators. This was the prevalence of any cardiovascular comorbidity by clustering six conditions: AF, CHF, PVD, MI, TIA and stroke.

5. Fifth, we fitted conditional logistic regression models (taking the matching set into account) to estimate OR and 95\% CI for associations between T2D and the 18 comorbidities. Regression models restricted to males or females were also fitted to calculate gender-specific estimates.

6. Sixth, using findings from analyses 1,2 and 5 , we developed multimorbidity metrics to indicate most prevalent morbidities in people with T2D (prevalence of $\geq 5 \%$ ) and/or morbidities highly associated with T2D diagnosis (OR of $\geq 2$ when compared with people without diabetes). We report these metrics in the overall cohort and by gender using the year 2014 cohort. We then assessed if these highly prevalent morbidities are featured in national diabetes guidelines.

All analyses were conducted using Stata Software: Release V.14(StataCorp LP).$^{25}$ 


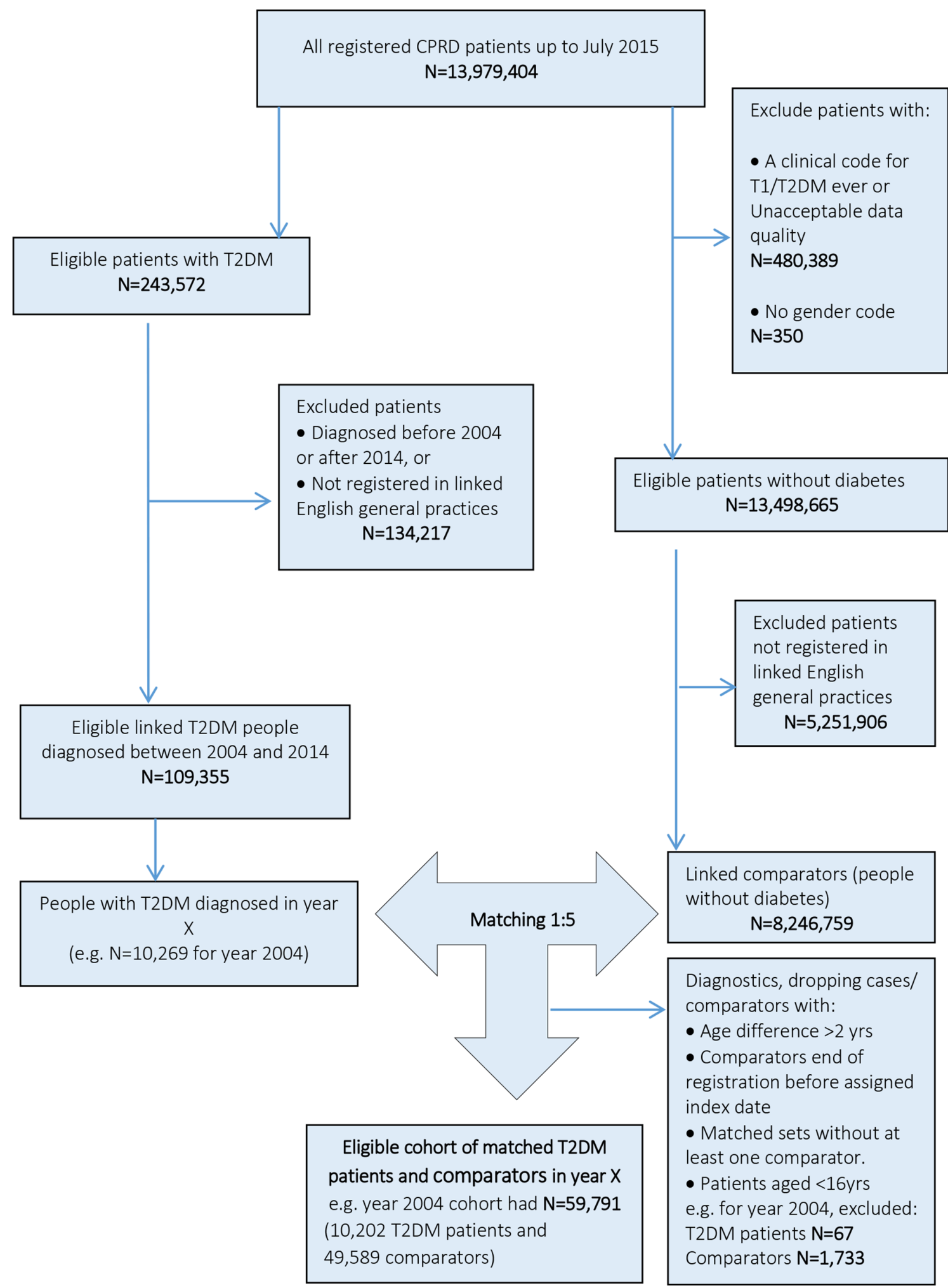

Figure 1 Flow chart for identification of patients with and without diabetes registered in linked general practices, using year 2004 as an example. CPRD, Clinical Practice Research Datalink; T2DM, type 2 diabetes mellitus.

\section{RESULTS}

\section{Patient population}

In total, 108588 people with T2D registered in 391 English general practices were included. Patients with T2D were matched to 528667 people without diabetes (comparators). A flow chart for identification of linked patients with T2D and comparators is illustrated in figure 1. The mean \pm SD preindex observation time for matched patients with T2D and comparators were similar throughout the study period and ranged between $3.7 \pm 3.7$ and $7.2 \pm 5.9$ years in patients with T2D and $3.7 \pm 3.6$ and $7.4 \pm 5.7$ years in comparators. 


\section{Comorbidities}

Annual prevalence of comorbidities

All comorbidities were more prevalent in people with T2D than the matched comparator patients (table 1, online supplementary table S2). Overall, $76.7 \%$ of patients with T2D and $54.2 \%$ of matched comparators without diabetes had at least one comorbidity. In 2014, $24 \%$ of patients with T2D vs $44 \%$ of comparators had none of the examined morbidities; $27 \%$ vs $24 \%$ had one morbidity; $20 \%$ vs $15 \%$ had two comorbidities; $14 \%$ vs $8 \%$ had three comorbidities, $7 \%$ vs $4 \%$ four comorbidities and $4 \%$ vs $2 \%$ had $\geq 5$ comorbidities (online supplementary figure S2). The prevalence rates of all 18 comorbidities in T2D and comparators in 2004 and 2014 are presented in figure 2. Hypertension was the most prevalent comorbidity in all patients. The prevalence of cardiovascular conditions and risk factors were up to twofold higher in people with T2D than in comparators. The prevalence of CKD increased sharply over the study period in people with and without diabetes (from $1.3 \%$ (95\% CI $1.1 \%$ to $1.6 \%$ ) in 2004 to $8.2 \%$ (95\% CI $8.0 \%$ to $8.5 \%$ ) in 2014 in patients with T2D; and from $0.6 \%$ (95\% CI $0.58 \%$ to $0.72 \%$ ) in 2004 to $5.4 \%$ (95\% CI $5.3 \%$ to 5.5\%) in 2014 in comparators). Epilepsy and schizophrenia were the least common chronic conditions in both people with and without diabetes.

Age-specific, gender-specific and IMD-level-specific prevalence of comorbidities

By age, the number of comorbidities in patients with T2D and comparators increased with increasing age. The mean number of comorbidities was higher in people with T2D than in comparators for all age groups and were higher in females than males (figure 3A). In people with T2D, age-specific prevalence rates of the most common comorbidities were estimated, showing an overall increasing prevalence with increasing age at diagnosis of T2D (online supplementary figure S3). However, the proportion of people with asthma was nearly the same across all age groups while depression was less prevalent in patients aged $\geq 56$ years.

By gender, in patients with T2D and comparators higher prevalence of comorbidities was observed in females than in males during all study years (20042014). Gender-specific rates in 2014 are presented in figure 3B. The prevalence of hypothyroidism was nearly four-times higher in females (ranged between 10.2\% and $11.2 \%$ in patients with T2D and between $6.5 \%$ and $8.0 \%$ in comparators) than in males (between $2.6 \%$ and $3.0 \%$ in T2D and between $1.5 \%$ and $1.9 \%$ in comparators). MI was more prevalent in males than females in both patients with T2D and comparators.

By social deprivation level, multimorbidity prevalence rates did not vary greatly across patient-level IMD quintiles (quintile 1: least deprived; quintile 5: most deprived). However, the prevalence rates for asthma, COPD, anxiety and depression in patients with T2D diagnosed in 2014 were highest among the most socially deprived patients (online supplementary figure S4). This trend was more profound with depression. A similar pattern of prevalence was observed in comparators matched on the same year, but at lower prevalence rates.

\section{Proportion of patients with two comorbidities}

The 10 most common two coexisting morbidities in people with T2D and people without diabetes in 2014 were combinations of hypertension, hyperlipidaemia and OA (online supplementary table S3). The proportion of patients with two comorbidities was higher in people with T2D compared with comparators, and increased between 2004 and 2014. The pattern of all two coexisting comorbidities in people with and without T2D in 2014 are illustrated in 3D bubble plots in figure $4 \mathrm{~A}, \mathrm{~B}$, respectively.

\section{Annual prevalence rates of cardiovascular disease}

The prevalence of clustered cardiovascular disease (clustered six conditions: AF, CHF, PVD, MI, stroke and TIA) in people with T2D was higher than in matched comparators. This trend was observed across the study period and remained consistent when the prevalence rates were further stratified by gender (online supplementary figure S5). Males were more likely to have a cardiovascular condition than females.

\section{Association of T2D with comorbidities}

T2D diagnosis was associated with significantly higher risk for all prevalent comorbidities except cancer. In comparison to people without diabetes (comparators) in 2014, patients with T2D were twice as likely as people without diabetes to have schizophrenia (OR 2.38, $95 \%$ CI 1.88 to 3.01 ) or a cardiovascular disease: CHF (OR 2.12, 95\% CI 1.84 to 2.43 ); PVD (OR 2.01, 95\% CI 1.68 to 2.40 ); MI (OR 2.13, 95\% CI 1.85 to 2.46) (online supplementary figure $\mathrm{S} 6$ ). ORs were overall stable when assessed over the study period between 2004 and 2014 (online supplementary table S4). In gender-specific analyses, the presence of T2D was associated with significantly higher risk for all 18 comorbidities in females (figure 5). Whereas, ORs for prevalent epilepsy and cancer were not statistically significant between male patients with T2D and comparators. T2D diagnosis was associated with higher risks for all comorbidities in women than in men. Similar estimates were obtained when regression models were also adjusted for IMD quintiles.

In addition to confirming the existence of some 'known' comorbidities in people with T2D such as hypertension, hyperlipidaemia, CVD and CKD, our metrics highlighted several other highly prevalent morbidities, namely, OA, hypothyroidism, asthma, COPD, anxiety, depression and schizophrenia (online supplementary table S5, online supplementary figure S7). By gender, hyperlipidaemia was more associated with T2D diagnosis in women than in men; while anxiety was more prevalent in men, using OR and prevalence rates, respectively. 


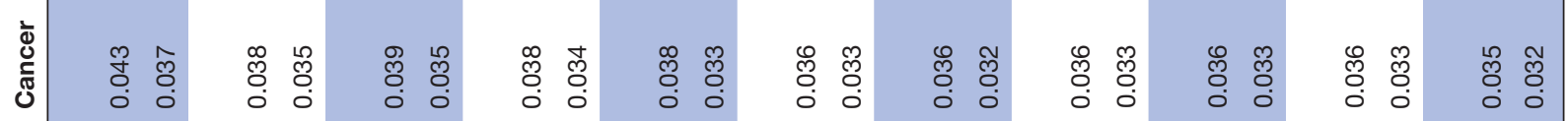

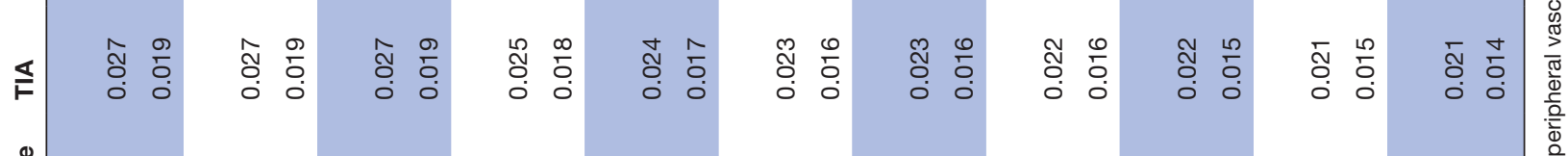

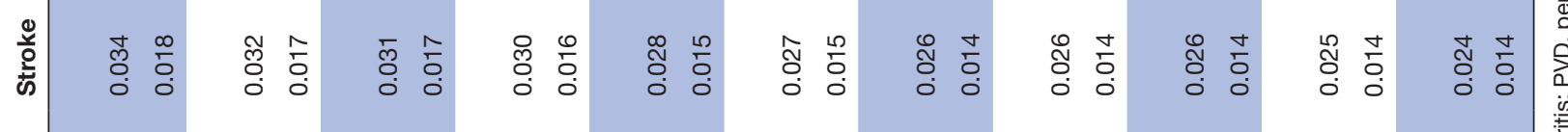

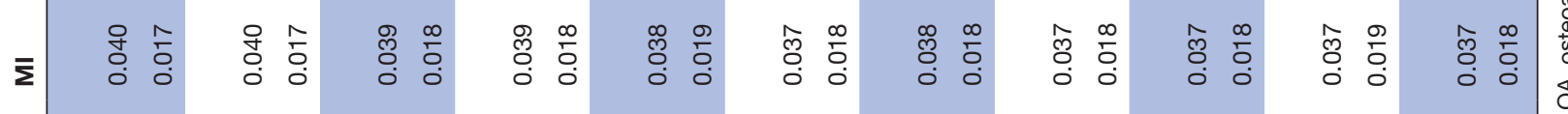

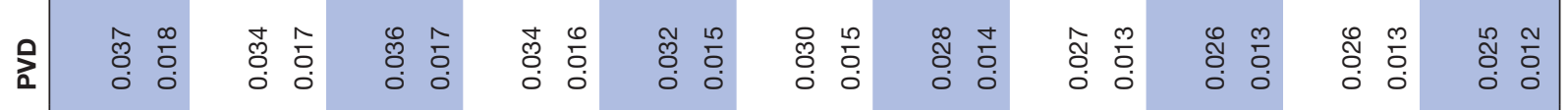

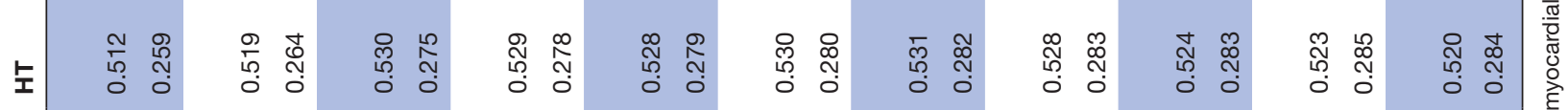

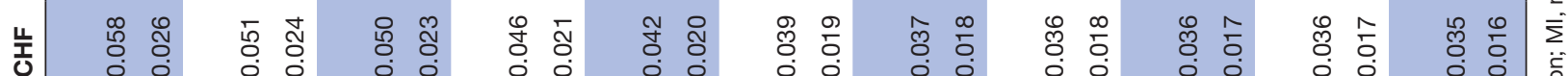

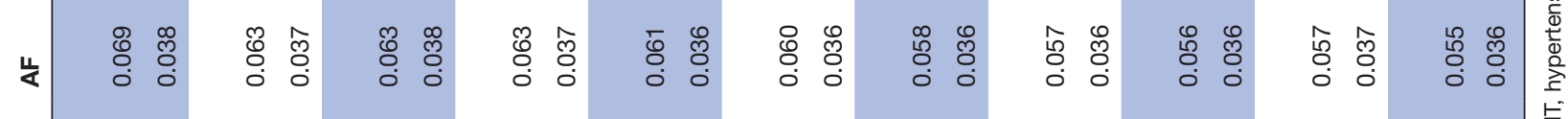

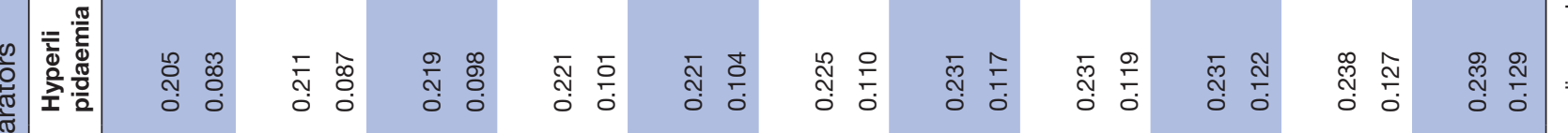
产

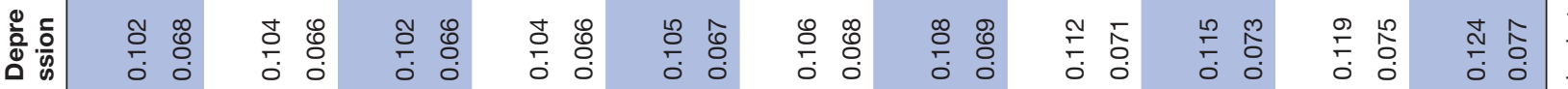
娄 0 i

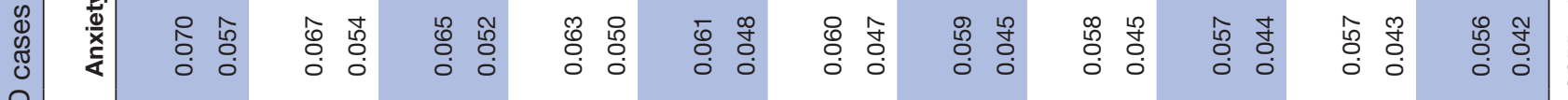

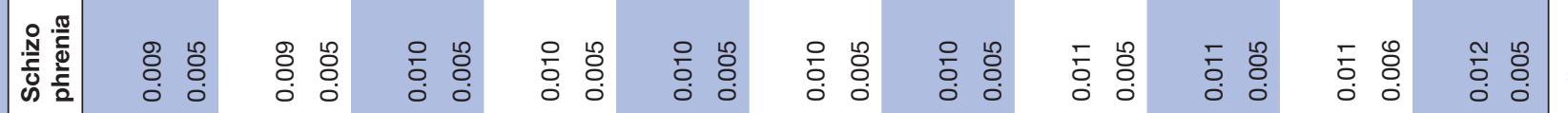
ปे

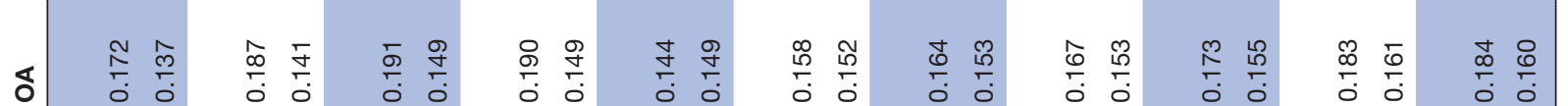

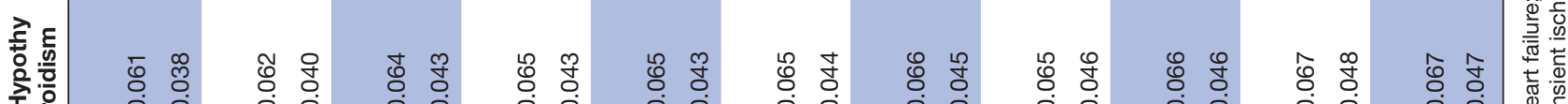

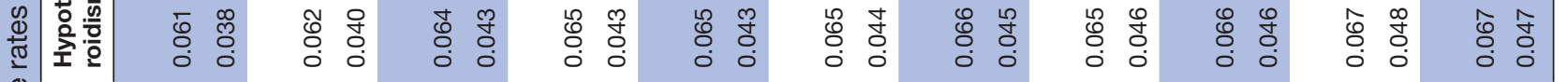

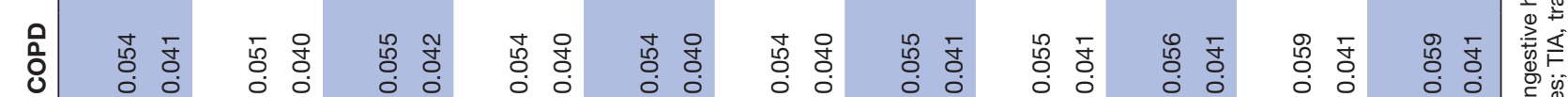

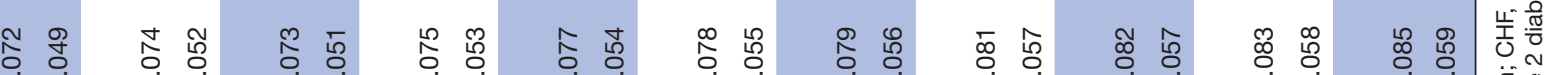

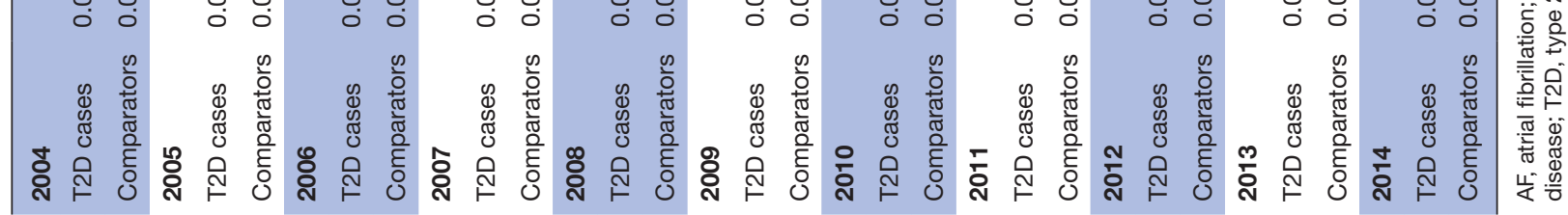




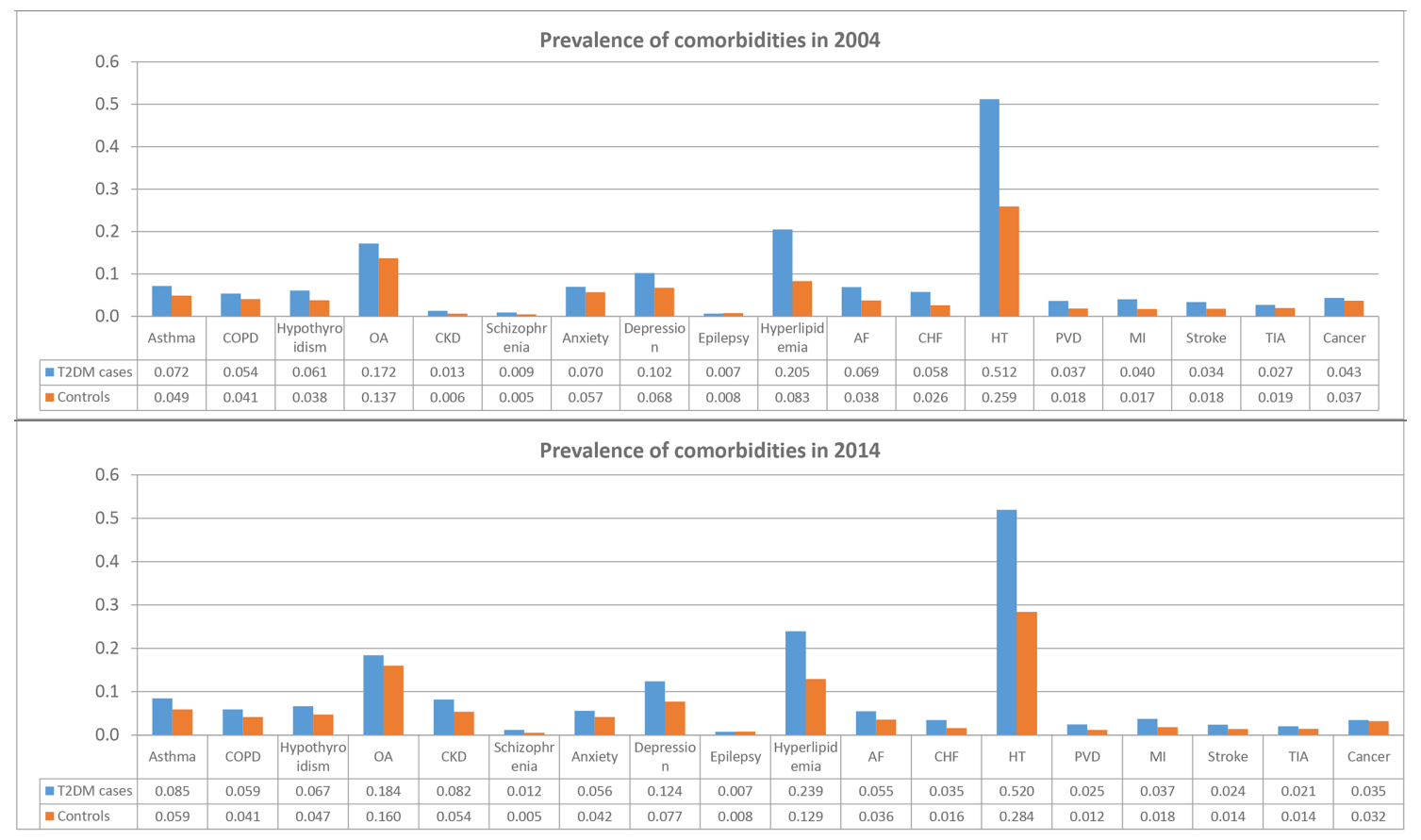

Figure 2 Prevalence of comorbidities in patients with type 2 diabetes mellitus (T2DM) and patients without diabetes in 2004 and 2014. AF, atrial fibrillation; CHF, congestive heart failure; CKD, chronic kidney disease; COPD, chronic obstructive pulmonary disease; HT, hypertension; OA, osteoarthritis; PVD, peripheral vascular disease; MI, myocardial infarction; TIA, transient ischaemic attack.

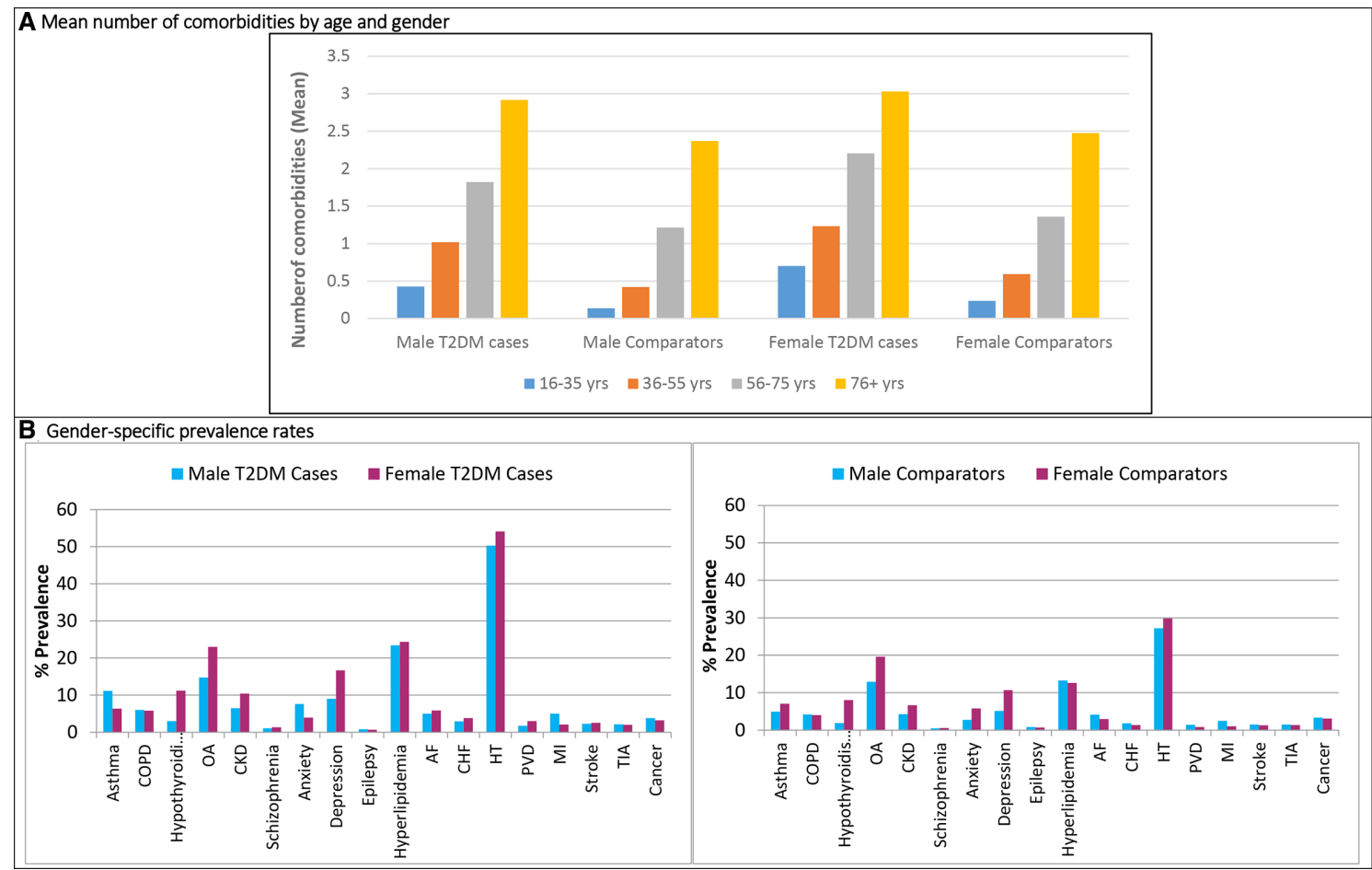

Figure 3 Trends of comorbidities in patients with type 2 diabetes (T2DM) and matched comparators without diabetes by age and gender in 2014. (A) Mean number of comorbidities in patients with T2DM and comparators by age categories and gender (B) gender-specific percentage prevalence of comorbidities at the study end 2014 in patients with T2DM and comparators without diabetes. AF, atrial fibrillation; $\mathrm{CHF}$, congestive heart failure; $\mathrm{CKD}$, chronic kidney disease; COPD, chronic obstructive pulmonary disease; HT, hypertension; OA, osteoarthritis; PVD, peripheral vascular disease; MI, myocardial infarction; TIA, transient ischaemic attack. 


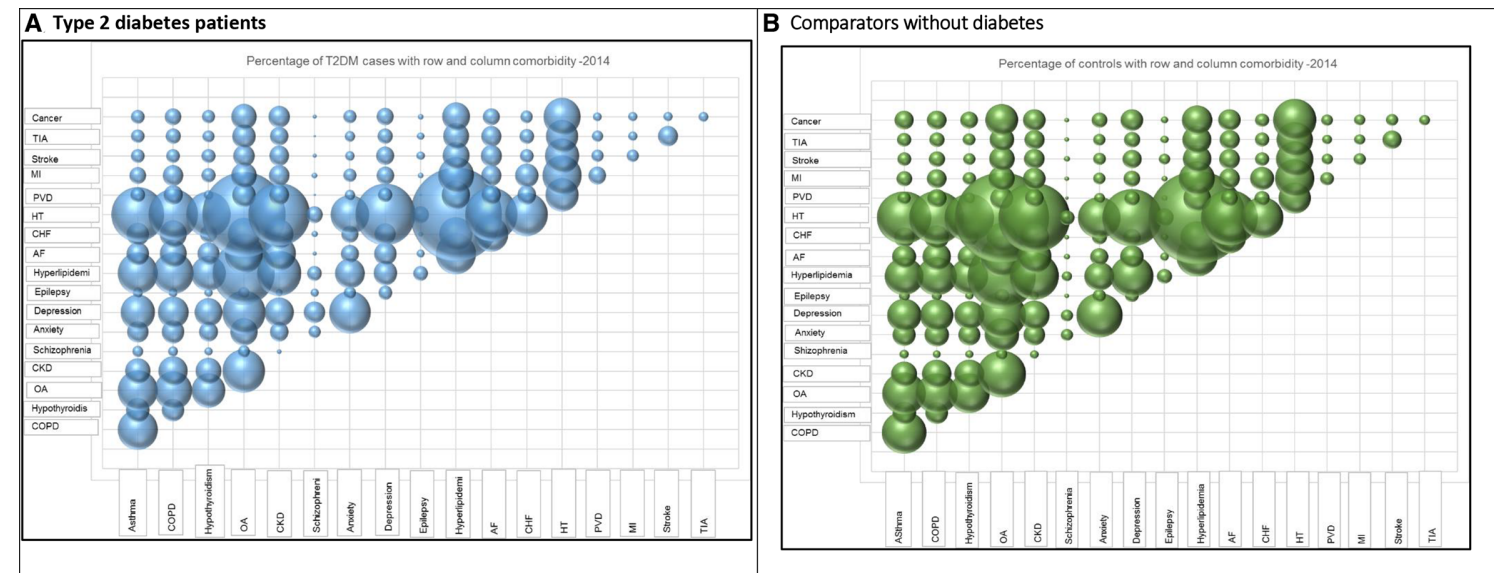

Figure 4 Percentage of patients with T2D mellitus (T2DM) and comparators with the row and the column comorbidity in 2014. $\mathrm{AF}$, atrial fibrillation; CHF, congestive heart failure; CKD, chronic kidney disease; COPD, chronic obstructive pulmonary disease; $\mathrm{HT}$, hypertension; OA, osteoarthritis; PVD, peripheral vascular disease; MI, myocardial infarction; TIA, transient ischaemic attack.

\section{DISCUSSION}

\section{Main findings}

To our best knowledge, this is the first large study in the UK to compare the patterns of 18 comorbidities between people with T2D and people without diabetes using primary and secondary care data. Our findings show that comorbidities were more prevalent in people with T2D than comparators throughout the 11-year study period. Nearly $77 \%$ of people with T2D had at least one comorbidity in comparison to $54 \%$ of people without diabetes. Overall, prevalence rates increased with age, comorbidities were more prevalent in women than in men, while it did not vary greatly by socioeconomic status except for depression and respiratory conditions where higher rates were observed in people from most deprived areas. People with T2D were twice as likely as those without diabetes to have a cardiovascular disease. Hypertension, in combination with hyperlipidaemia or $\mathrm{OA}$, was the most commonly coexisting morbidities in people with T2D. Importantly, our findings highlight other underreported comorbidities in people with T2D that are highly prevalent or associated with T2D diagnosis including schizophrenia, anxiety and OA.

\section{Findings in relation to literature}

Despite the metabolic complexity of T2D, most of past studies focused on cardiovascular comorbidities with little

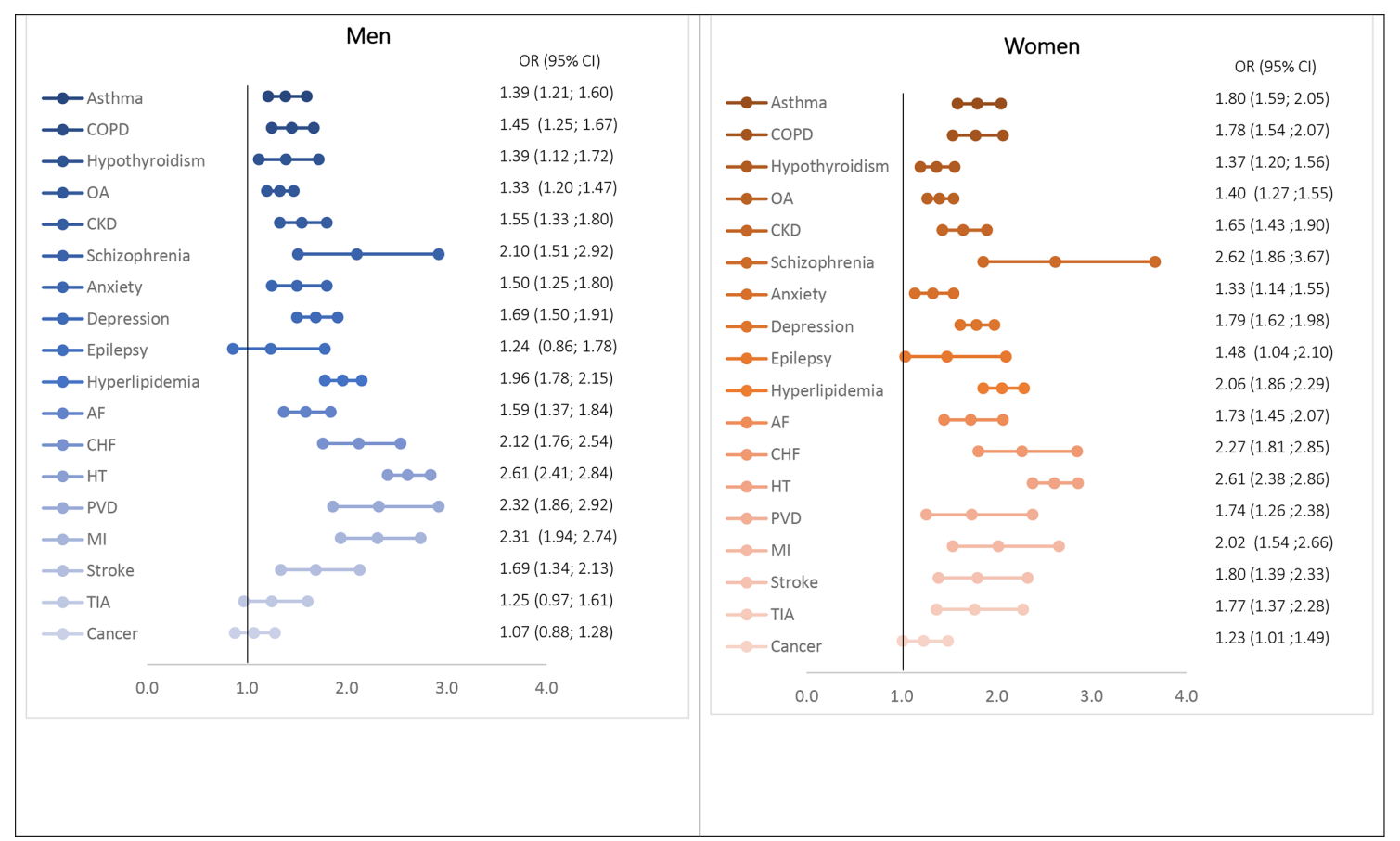

Figure 5 ORs for association of type 2 diabetes with comorbidities by gender in 2014. AF, atrial fibrillation; CHF, congestive heart failure; CKD, chronic kidney disease; COPD, chronic obstructive pulmonary disease; OA, osteoarthritis; PVD, peripheral vascular disease; MI, myocardial infarction; TIA, transient ischaemic attack. 
information on a wider consideration of physical and mental health illnesses, with limited studies based on UK data. However, reports based on non-UK population are available. ${ }^{26} 27$

Two notable studies have estimated that between $44 \%$ and $85 \%$ of people with diabetes have one or more comorbidity. ${ }^{1314}$ This is in agreement with our findings where $77 \%$ of patients with T2D had at least one comorbidity.

The first of these studies used primary and secondary care data in the Netherlands from 7499 people with diabetes of any type and assessed the impact of 10 comorbidities on healthcare utilisation (annual numbers of general practice visits, prescriptions and hospital admissions). ${ }^{13}$ The study reported that $44 \%$ of people with diabetes had $\geq 1$ comorbidity and that healthcare utilisation was strongly correlated with the number of diabetes-related and non-diabetesrelated comorbidities. The study provides important findings on the number and impact of multimorbidity in people with diabetes. However, our study cohort was larger $(\mathrm{n}=108588)$, included a comparator group, we examined more prevalent coexisting conditions and focused on people with T2D that differ from also including people with other forms of diabetes with different patterns of comorbidities (mainly type 1 diabetes).

A second study was exploratory and included 714 patients with newly diagnosed T2D between 1985 and 2007 registered in four general practices and examined a wide range of comorbidities at and after the time of diagnosis. ${ }^{14}$ The study found that $85 \%$ of patients had $\geq 1$ chronic comorbid condition at the time of diabetes diagnosis, and $25 \%$ of those without any comorbid condition at diagnosis developed $\geq 1$ comorbidity within the first year after diagnosis.

Other studies have reported on the prevalence of comorbidities in people with T2D, but mainly as a secondary research aim. Many of these studies had small sample sizes $^{8928}$; were based on a subgroup of patients with T2D, for example, within a prespecified age category ${ }^{10}{ }^{11}$; the examined comorbid diseases were either reported as an overall count of conditions ${ }^{9}$ or limited to diabetes-related diseases, mainly diabetes complications. ${ }^{12}{ }^{29-31}$ Populationbased studies based on routinely collected data to examine multimorbidity are limited and urgently needed. ${ }^{732}$

We showed that cardiovascular conditions or risk factors, such as hyperlipidaemia and hypertension, were particularly common in people with T2D as reported previously by the American Diabetes Association. ${ }^{1}$ We found that the prevalence of individual and clustered cardiovascular diseases was twice as high as in people without diabetes. This is an important finding as it helps to explain previous reports that cardiovascular disease is the main cause of increased morbidity and mortality in people with T2D. ${ }^{133}$ The observed increased prevalence of CKD from 2006 reflects the improved capture of the condition in UK primary care data attributed to the change in coding system, following its addition to the QOF domains on the same year, ${ }^{34} 35$ and a National Health Service (NHS) England mandate for laboratories to report estimated glomerular filtration rate (eGFR). The rates then become overall stable until the end of the study period in 2014. The higher rates of CKD in women may be partly explained by higher blood pressure levels and lower ACE inhibitor prescribing in women with T2D compared with men with T2D. ${ }^{36}$ We envisage that the observed overall higher rates of comorbid conditions in women may also be partially explained by women consulting primary care more than men in the UK, ${ }^{37}$ which may explain higher detection and subsequent diagnoses rates for some conditions. In consistency with our findings, a US study found that people with diabetes are at a greater risk for asthma and COPD than people without diabetes. ${ }^{38}$

\section{Strengths and limitations}

Our study has several strengths. First, to our knowledge, this study presents a novel analysis examining and comparing the patterns of multimorbidity using different approaches in a large population of people with T2D and people without diabetes using routine primary and secondary care data. Second, our large cohort provides sufficient statistical power to calculate overall and age, gender and IMD-specific prevalence rates of individual comorbidities. The selected 11-year study period is relevant to the clinical practice scheme ranging from QOF introduction in 2004 and up to 2014. Third, our study design of matching our cohorts on important confounders aimed to minimise bias. Fourth, the study used linked hospitalisation (HES) data, in addition to primary care data recorded by general practitioners, which has improved the capture of comorbidities diagnosed in secondary care setting. The added value of using linked data has been acknowledged in improving the validity of research studies, where, for example data linkage to secondary care data has enriched the identification of examined conditions as reported previously with cardiovascular disease ${ }^{3940}$ Fifth, to minimise misclassification, we examined prescribed treatments and time-windows as additional measures to confirm the active status of five resolvable medical conditions, such as depression and asthma. Finally, besides the 'known' and frequently reported comorbidities such as cardiovascular disease, our findings highlight a number of comorbidities that are highly prevalent in people with T2D but underreported in national diabetes guidelines.

The study also has some limitations, which are important to consider when interpreting the findings. First, this study relies on recorded diagnoses in electronic health records and therefore there is a possibility to have missed patients with undiagnosed illnesses such as patients who did not consult general practice or hospital setting. Also, we have not included other possible conditions, such as dementia and gastrointestinal conditions, ${ }^{41}$ but we aimed to examine the most clinically relevant and well-recorded morbidities to minimise misclassification. Second, the observed increased CKD prevalence should be interpreted carefully as it mainly reflects the improved capture of the condition in primary care data in later years of the study that is, a resolved data recording issue. Third, given the use of an inception cohort, the duration of diabetes was limited and is likely to have led to some underestimation of the burden of multimorbidity 
in all community-based people with T2D. Although studies of people with prevalent diabetes would also be valuable in future research, our design to include a cohort of incident patients aimed to reduce time-dependent biases related to the timing of T2D diagnosis between included patients in terms of T2D severity and survival time.

\section{Clinical implications}

With the increased longevity after T2D diagnosis, ${ }^{5}$ multimorbidity in people with diabetes is a major public health issue with an economic burden affecting heath service utilisation. People with multimorbidity are prone to premature death, higher risk of hospital admissions and poorer quality of life. ${ }^{6}$ In addition, the pathophysiological complexity and multiorgan involvement of T2D drives the need to investigate the patterns of coexisting conditions. The challenge of multimorbidity is further compounded by the fact that most available clinical guidelines are designed as syndromefocused guidance without considering multimorbidity. ${ }^{42}$ In 2016, the first UK clinical guidance on managing multiple long-term conditions in adults was released with its main recommendations focussing on implementing a patienttailored approach to care and involving the patient in decisions around the chosen model of care. ${ }^{43}$ Yet, many other clinical guidelines for the management of diabetes do not consider multimorbidity which may affect their clinical utility. $^{14}$

Defining patterns of multimorbidity can have important clinical applications such as identifying potentially harmful drug-disease interactions, ${ }^{44}$ establishing optimal targeting of specific therapies, and implementing individualised management plans around the time of diagnosis. A better understanding of multimorbidity could help clinicians to design and provide comprehensive care pathways (ie, more holistic rather than condition-specific care) and to action primary and secondary prevention of comorbidities in people with T2D. This could eventually improve patients' quality of life and minimise healthcare utilisation and associated costs.

Our results of mapped multimorbidity burden in people with T2D highlight the main morbidities associated with T2D diagnosis, mainly CVD and mental health illnesses. We also provide these trends by patient demographic variables, which further identifies groups of people with T2D with higher prevalence (by age, gender, social deprivation), which can support clinicians and other healthcare professional teams (including nurses and pharmacists) identify people at greater risk for developing these morbidities and to provide holistic care plans. Our results can have important clinical and public health implications, including:

1. Our results show that the prevalence of depression in people with T2D aged 16-35 years were higher compared with rates observed in people aged 56 and over (online supplementary figure S3). This finding highlights a profound clinical need in young people who could benefit enormously from mental health intervention.

2. Multimorbidity is associated with a higher number of prescribed medications (polypharmacy). Mapping the patterns of multimorbidity can therefore help guide to develop more comprehensive care plans. For instance, this mapping could help avoid potential drug-disease interactions such as interactions between some second-line diabetes therapies and CKD or heart failure.

3 . We showed that the association of comorbidities with T2D diagnosis was generally higher in women compared with men. This suggests a need for future studies to investigate the reasons for these differences, which may inform future gender-specific multimorbidity prevention and management strategies.

4. Identifying the trends of the proportion of people having two coexisting conditions, as illustrated in figure 4, provides an insight into the development of physical and mental morbidities in people with T2D which could inform future prevention strategies. This finding addresses the current need for more research on the coexistence of physical and mental illnesses as highlighted in the recent Academy of Medical Sciences multimorbidity report. ${ }^{745}$

5. Our findings highlight the need for future clinical guidelines to refocus patient-centred care in T2D on non-cardiometabolic conditions such as asthma, COPD, anxiety, depression, schizophrenia and OA.

6 . We report the prevalence of comorbidities in the context of social deprivation, which has potential to inform future public health policy. This addresses a reported need to understand the link between multimorbidity and socioeconomic deprivation while considering policy and practice responses to multimorbidity. ${ }^{46}$

Our new multimorbidity metric showed that some of the most prevalent morbidities have not featured in national diabetes guidelines including OA, hypothyroidism, asthma, COPD, anxiety and schizophrenia. The latter are discordant conditions (ie, they have a different pathophysiology compared with diabetes) that are rarely covered in clinical guidance unlike concordant comorbidities. ${ }^{47} 48$ Importantly, we found that several national diabetes guidelines in the UK, Canada and the USA do not address several of the highly prevalent comorbidities on our multimorbidity metrics including $\mathrm{OA}$, anxiety, schizophrenia, asthma and COPD. ${ }^{49-51}$ In light of this important finding, it is the time for national diabetes guidelines developers to reassess the 'single-condition' focus of current guidelines and step further from limiting recommendations to only a few comorbidities (eg, CVD) in people with T2D. Future guidelines should provide actionable and integrated recommendations for clinical practice to better manage increasing multimorbidity burden in people with T2D considering mental illnesses and other discordant comorbidities that are becoming more prevalent in recent years.

\section{CONCLUSION}

In conclusion, we provide the first detailed 11-year epidemiological analysis of multimorbidity burden in people with T2D in comparison with individuals without diabetes using linked national primary care and hospital data. Our 
analysis involved a wide spectrum of under-reported comorbidities and showed a higher prevalence of all examined physical and mental health comorbidities in people with T2D. Comorbidities were higher in people with T2D than comparators, increased with age and were more prevalent in women compared with men. In addition to cardiovascular conditions, our findings show that among the highly prevalent comorbidities are discordant physical and mental health conditions not broadly reported in literature and UK and other national diabetes guidelines. Our study is clinically relevant and can have important clinical and public health implications and inform the development of realistic patient-centred care plans for people with recently diagnosed T2D in the context of multimorbidity. Our findings indicate that future national guidelines need to provide practical recommendations and models for diabetes care delivery while considering a wider range of comorbidities.

\section{Author affiliations}

${ }^{1}$ Centre for Primary Care and Health Services Research, Division of Population Health, Health Services Research and Primary Care, School of Health Sciences, Faculty of Biology, Medicine and Health, Manchester Academic Health Science Centre (MAHSC), University of Manchester, Manchester, UK

${ }^{2}$ Department of Pharmaceutics, Faculty of Pharmacy, University of Tripoli, Tripoli, Libya

${ }^{3}$ Centre for Pharmacoepidemiology and Drug Safety, Division of Pharmacy and Optometry, School of Health Sciences, Faculty of Biology, Medicine and Health, Manchester Academic Health Sciences Centre (MAHSC), University of Manchester, Manchester, UK

${ }^{4}$ Division of Diabetes, Endocrinology and Gastroenterology, School of Medical Sciences, Faculty of Biology, Medicine and Health, Manchester Academic Health Science Centre (MAHSC), University of Manchester, Manchester, UK ${ }^{5}$ Diabetes, Endocrinology and Metabolism Centre, Manchester University NHS Foundation Trust, Manchester Academic Health Science Centre (MAHSC), Manchester, UK

Acknowledgements SSZ would like to thank the Libyan Ministry for Higher Education for the scholarship provided for her $\mathrm{PhD}$ programme during this study. This study is based on data from the CPRD obtained under license from the UK Medicines and Healthcare products Regulatory Agency (MHRA). The data are provided by patients and collected by the NHS as part of their care and support. Hospital Episode Data Copyright (2015) are reused with the permission of The Health and Social Care Information Centre. All rights reserved. The interpretation and conclusions contained in this paper are those of the authors alone. The study protocol was approved by CPRD's Independent Scientific Advisory Committee (ISAC) (reference: 15_149). Parts of this study were presented at the Diabetes UK Professional Conference, Manchester, UK, March 2017.

Contributors SSZ, DS, MKR and DA contributed to the study protocol. DS, DA and SSZ contributed to the study design. SSZ extracted and analysed the data and drafted the initial draft of the manuscript. SSZ had full access to all study data, performed all the statistical analyses, supervised by DS and DA and takes responsibility for the integrity of the data and the accuracy of data analyses. All authors contributed to interpretation of data and revised the paper for important intellectual content and agreed on the final version before submission.

Funding The authors have not declared a specific grant for this research from any funding agency in the public, commercial or not-for-profit sectors.

Competing interests MKR has received educational grant support from MSD and Novo Nordisk; has modest stock ownership in GSK; and has consulted for Roche. DMA reports research grants from Abbvie, Almirall, Celgene, Eli Lilly, Novartis, UCB and the Leo Foundation.

Patient and public involvement Patients and/or the public were not involved in the design, or conduct, or reporting, or dissemination plans of this research.

Patient consent for publication Not required.
Ethics approval The study was approved by the Independent Scientific Advisory Committee (ISAC) for MHRA Database Research (protocol number: 15_149). Generic ethical approval for observational research using the Clinical Practice Research Datalink (CPRD) with approval from ISAC has been granted by a Health Research Authority (HRA) Research Ethics Committee (East Midlands-Derby, REC reference number 05/MRE04/87).

Provenance and peer review Not commissioned; externally peer reviewed.

Data availability statement Data may be obtained from a third party and are not publicly available. Access to data is available only once approval has been obtained through the Clinical Practice Research Datalink. All data relevant to the study are included in the article or uploaded as supplementary information. Electronic health records are, by definition, considered sensitive data in the UK by the Data Protection Act and cannot be shared via public deposition because of information governance restriction in place to protect patient confidentiality.

Open access This is an open access article distributed in accordance with the Creative Commons Attribution Non Commercial (CC BY-NC 4.0) license, which permits others to distribute, remix, adapt, build upon this work non-commercially, and license their derivative works on different terms, provided the original work is properly cited, appropriate credit is given, any changes made indicated, and the use is non-commercial. See: http://creativecommons.org/licenses/by-nc/4.0/.

\section{ORCID iD}

Salwa S Zghebi http://orcid.org/0000-0002-7978-1094

\section{REFERENCES}

1 Standards of medical care in diabetes--2015: summary of revisions. Diabetes Care 2015;38:S4.

2 World Health Organization (WHO) media centre. Diabetes. secondary diabetes [Fact sheet], 2016. Available: http://www.who.int/ mediacentre/factsheets/fs312/en/

3 Lind M, Garcia-Rodriguez LA, Booth GL, et al. Mortality trends in patients with and without diabetes in Ontario, Canada and the UK from 1996 to 2009: a population-based study. Diabetologia 2013;56:2601-8.

4 Mulnier HE, Seaman HE, Raleigh VS, et al. Mortality in people with type 2 diabetes in the UK. Diabet Med 2006;23:516-21.

5 Zghebi SS, Steinke DT, Carr MJ, et al. Examining trends in type 2 diabetes incidence, prevalence and mortality in the UK between 2004 and 2014. Diabetes Obes Metab 2017;19:1537-45.

6 Smith SM, Soubhi H, Fortin M, et al. Managing patients with multimorbidity: systematic review of interventions in primary care and community settings. BMJ 2012;345:e5205.

7 The Academy of Medical Sciences. Multimorbidity: a priority for global health research, 2018.

8 Ko J, Delafield R, Davis J, et al. Characteristics of patients with type 2 diabetes mellitus in two rural, medically underserved communities. Hawaii J Med Public Health 2013;72:191-6.

9 Chiu C-J, Wray LA. Factors predicting glycemic control in middleaged and older adults with type 2 diabetes. Prev Chronic Dis 2010;7:A08.

10 Carnethon MR, Biggs ML, Barzilay Jl, et al. Longitudinal association between depressive symptoms and incident type 2 diabetes mellitus in older adults: the cardiovascular health study. Arch Intern Med 2007;167:802-7.

11 Asche CV, McAdam-Marx C, Shane-McWhorter L, et al. Evaluation of adverse events of oral antihyperglycemic monotherapy experienced by a geriatric population in a real-world setting: a retrospective cohort analysis. Drugs Aging 2008;25:611-22.

12 Bo S, Castiglione A, Ghigo E, et al. Mortality outcomes of different sulphonylurea drugs: the results of a 14-year cohort study of type 2 diabetic patients. Eur J Endocrinol 2013;169:117-26.

13 Struijs JN, Baan CA, Schellevis FG, et al. Comorbidity in patients with diabetes mellitus: impact on medical health care utilization. BMC Health Serv Res 2006;6:84.

14 Luijks H, Schermer T, Bor H, et al. Prevalence and incidence density rates of chronic comorbidity in type 2 diabetes patients: an exploratory cohort study. BMC Med 2012;10:128.

15 Medicines \& Healthcare products Regulatory Agency (MHRA). Clinical practice research datalink. secondary clinical practice research datalink, 2018. Available: https://www.cprd.com/home/

16 Herrett E, Gallagher AM, Bhaskaran K, et al. Data resource profile: clinical practice research datalink (CPRD). Int J Epidemiol 2015;44:827-36.

17 Williams T, van Staa T, Puri S, et al. Recent advances in the utility and use of the general practice research database as an example of a UK primary care data resource. Ther Adv Drug Saf 2012;3:89-99. 
18 Department for Communities and Local Government. The english indices of deprivation 2010. secondary the english indices of deprivation 2010, 2011. Available: https://www.gov.uk/government/ uploads/system/uploads/attachment data/file/6871/1871208.pdf

19 Tonelli M, Wiebe N, Fortin M, et al. Methods for identifying 30 chronic conditions: application to administrative data. BMC Med Inform Decis Mak 2015;15:31.

20 Quan H, Sundararajan V, Halfon P, et al. Coding algorithms for defining comorbidities in ICD-9-CM and ICD-10 administrative data. Med Care 2005;43:1130-9.

21 Springate DA, Kontopantelis E, Ashcroft DM, et al. Clinical codes: an online clinical codes repository to improve the validity and reproducibility of research using electronic medical records. PLOS One 2014;9:e99825.

22 World Health Organization (WHO) International statistica classification of diseases and related health problems 10th revision (ICD-10). secondary international statistical classification of diseases and related health problems 10th revision (ICD-10), 2015. Available: http://apps.who.int/classifications/icd10/browse/2015/en

23 Lester H, Campbell S. Developing quality and outcomes framework (QOF) indicators and the concept of 'QOFability'. Qual Prim Care 2010;18:103-9

24 Cooper S-A, McLean G, Guthrie B, et al. Multiple physical and mental health comorbidity in adults with intellectual disabilities: population-based cross-sectional analysis. BMC Fam Pract 2015;16:110.

25 StataCorp LLC. Stata statistical software. secondary stata statistical software, 2018. Available: https://www.stata.com/

26 Trento M, Trevisan M, Raballo M, et al. Depression, anxiety, cognitive impairment and their association with clinical and demographic variables in people with type 2 diabetes: a 4-year prospective study. $J$ Endocrinol Invest 2014:37:79-85

27 Alonso-Morán E, Orueta JF, Fraile Esteban JI, Esteban JIF, et al. The prevalence of diabetes-related complications and multimorbidity in the population with type 2 diabetes mellitus in the Basque country. BMC Public Health 2014;14:1059.

28 Nefs G, Pop VJM, Denollet J, et al. The longitudinal association between depressive symptoms and initiation of insulin therapy in people with type 2 diabetes in primary care. PLoS One 2013;8:e78865.

29 Riedel AA, Heien $\mathrm{H}$, Wogen J, et al. Loss of glycemic control in patients with type 2 diabetes mellitus who were receiving initial metformin, sulfonylurea, or thiazolidinedione monotherapy. Pharmacotherapy 2007;27:1102-10.

30 Shah S, Das AK, Kumar A, et al. Baseline characteristics of the Indian cohort from the improve study: a multinational, observational study of biphasic insulin aspart 30 treatment for type 2 diabetes. Adv Ther 2009;26:325-35.

31 Sharma SK, Seshiah V, Sahay BK, et al. Baseline characteristics of the improve control study population: a study to evaluate the effectiveness of a standardized healthcare professionals training program. Indian J Endocrinol Metab 2012;16:S471-3.

32 France EF, Wyke S, Gunn JM, et al. Multimorbidity in primary care: a systematic review of prospective cohort studies. Br J Gen Pract 2012;62:e297-307.

33 National Collaborating Centre for Chronic Conditions. Type 2 diabetes: national clinical guideline for management in primary and secondary care (update). secondary type 2 diabetes: national clinical guideline for management in primary and secondary care (update), 2008. Available: http://www.nice.org.uk/nicemedia/live/11983/40803/ 40803.pdf
34 Nihat A, de Lusignan S, Thomas N, et al. What drives quality improvement in chronic kidney disease (CKD) in primary care: process evaluation of the quality improvement in chronic kidney disease (QICKD) trial. BMJ Open 2016;6:e008480.

35 Health and Social Care Information Centre. Quality and outcomes framework. secondary quality and outcomes framework. Available: http://www.hscic.gov.uk/qof

36 Wright AK, Kontopantelis E, Emsley R, et al. Cardiovascular risk and risk factor management in type 2 diabetes mellitus. Circulation 2019;139:2742-53.

37 Wang Y, Hunt K, Nazareth I, et al. Do men consult less than women? an analysis of routinely collected UK general practice data. BMJ Open 2013;3:e003320.

38 Ehrlich SF, Quesenberry CP, Van Den Eeden SK, et al. Patients diagnosed with diabetes are at increased risk for asthma, chronic obstructive pulmonary disease, pulmonary fibrosis, and pneumonia but not lung cancer. Diabetes Care 2010;33:55-60.

39 Herrett E, Shah AD, Boggon R, et al. Completeness and diagnostic validity of recording acute myocardial infarction events in primary care, hospital care, disease registry, and national mortality records: cohort study. BMJ 2013;346:f2350.

40 Padmanabhan S, Carty L, Cameron E, et al. Approach to record linkage of primary care data from clinical practice research datalink to other health-related patient data: overview and implications. Eur $J$ Epidemiol 2019;34:91-9.

41 Barnett K, Mercer SW, Norbury M, et al. Epidemiology of multimorbidity and implications for health care, research, and medical education: a cross-sectional study. Lancet 2012;380:37-43.

42 Guthrie B, Payne K, Alderson P, et al. Adapting clinical guidelines to take account of multimorbidity. BMJ 2012;345:e6341.

43 National Institute for Health and Care Excellence. Multimorbidity: clinical assessment and management. secondary multimorbidity: clinical assessment and management, 2016. Available: nice.org.uk/ guidance/ng56

44 Dumbreck S, Flynn A, Nairn M, et al. Drug-disease and drug-drug interactions: systematic examination of recommendations in 12 UK national clinical guidelines. BMJ 2015;350:h949.

45 Stirland LE, González-Saavedra L, Mullin DS, et al. Measuring multimorbidity beyond counting diseases: systematic review of community and population studies and guide to index choice. BMJ 2020;368:m160.

46 The Richmond Group of Charities. Multimorbidity - understanding the challenge. secondary multimorbidity - understanding the challenge, 2018. Available: https://richmondgroupofcharities.org.uk/ sites/default/files/multimorbidity - understanding the challenge.pdf

47 Piette JD, Kerr EA. The impact of comorbid chronic conditions on diabetes care. Diabetes Care 2006;29:725-31.

48 Tran J, Norton R, Conrad N, et al. Patterns and temporal trends of comorbidity among adult patients with incident cardiovascular disease in the UK between 2000 and 2014: a population-based cohort study. PLoS Med 2018;15:e1002513.

49 National Institute for Health and Clinical Excellence (NICE). Type 2 diabetes in adults: management(NICE guideline 28). Secondary type 2 diabetes in adults: management (NICE guideline 28), 2015 Available: https://www.nice.org.uk/guidance/ng28

50 Canadian Diabetes Association. Canadian diabetes association 2008 clinical practice guidelines for the prevention and management of diabetes in Canada. Can J Diabetes 2008;32.

51 American Diabetes Association. Comprehensive medical evaluation and assessment of comorbidities: standards of medical care in diabetes-2018. Diabetes Care 2018;41:S28-37. 